\title{
How fast is the distance between Earth and Sun changing in the Solar System?
}

\author{
Jim Henry ${ }^{1}$, Mesut Yurukcu ${ }^{2 *}$ and George Nnanna ${ }^{2}$ \\ a Henry Resources, Midland, TX, 79703 \\ b University of Texas Permian Basin, College of Engineering, Midland, TX, 79705 \\ * Correspondence: yurukcu_m@utpb.edu \\ Highlights \\ The cosmological expansion and acceleration of local systems such as Solar \\ System, \\ Calculation of the speed between Earth and Sun, \\ The acceleration of the Earth and Sun is $3.2 \times 10^{-10} \frac{\mathrm{m}}{\mathrm{s}}$,
}

\begin{abstract}
This paper aims to investigate the rate of expansion and extraction within the solar system. We carried out the Solar system expansion calculations to do such a review. The Universe is expected to look the same from every point in it. After the big bang, Universe is expanding at some speed. Astrophysicists have been in a race to measure precisely how fast the Universe is expanding since Hubble announced that galaxies were systematically moving away from Milky Way Galaxy with a current speed in 1929. Hubble's observations came after Einstein's general relativity, which inspired the big bang theory. According to the Big Bang theory, the Universe has created billions of years ago with an explosion and started to expand until today. The expansion of the Universe mostly happens in vast spaces, so clusters of galaxies move away from each other. For example, raising bread during baking will expand, but the raisings will stay the same size while moving each other to expand the bread. Observers have proven that an object (galaxies, a cluster of planets) held together by gravity has a patch of nonexpanding space produced by a gravitational field. However, some observers claimed the solar system is not expanding, while others claimed it is expanding. Does our solar system expand in an expanding Universe? The cosmological expansion of local systems is reviewed in the modern cosmological models. We showed answers related to this question with the help of literature. This review article revisited the proof of the Solar System's expansion and its speed with about $0.32 \frac{\mathrm{nm}}{\mathrm{s}}$ in an expanding Universe.
\end{abstract}

Keywords; Solar System; Earth; Sun; Expansion; Universe

\section{1-. Introduction}

In a clear sky, we see the Universe at rest with all of the stars and planets from our planet during the night. Outside of the Earth, the space is empty, and the stars dispersed light-years apart. To understand the Universe, we search by natural laws to understand how it governs the Universe. Albert Einstein described the Universe on an enormous imaginable scale with his general relativity theory. Thus, the Universe's fate became imaginable for theoretical scientific exploration. In 1929, Edwin Hubble predicted general 
relativity and discovered the expansion of the Universe. After Hubble's discovery about the relationship between the galaxies' distances and motions, Cosmologists and astrophysicists started a race for measuring precisely the Universe's expansion.

There are many common misconceptions about what happens as the Universe expands. Space expansion, as time goes called Universe expansion. The Universe carries the galaxies during its expansion. On the other side, photons' wavelength expands while photons are traveling through space. We see the red and blue shift from the galaxies far away from our galaxy once we look at the data. When a galaxy moves away from our galaxy, a photon that travels through space arrives in the Earth. While a photon travels through space, the photon's wavelength expands with the expansion of the Universe. Our eyes see this expand wavelength as a red color. If the photon travels a longer distance, the wavelength of the photon will become wider. Thus, photons coming from distant galaxies have larger redshifts called cosmological redshifts.

On the contrary, if the photons come from the closing stars-galaxies, we see the photons as blue. It is different from a Doppler shift. An object's motion through space caused doppler shifts, while space expansion causes a cosmological redshift. This is because space expands with force, pushing objects apart due to the cosmological constant's effect. However, the matter was floating in the exceedingly early Universe because of cosmic inflation.

Moreover, gravity causes the local clumping of matter into stars and galaxies. The neighboring objects' separation and Universe expansion's inertia are the same in a vacuum environment. Objects form and bond by gravity and no longer move away from each other-an explosion of matter through the vacuum environment described by the geometry that expands the whole Universe. Einstein's General Relativity predicts the light-photonsmove at the " $\mathrm{c}$ " speed for the exploding matter's local motion. Traveling the light situation changes with the dark energy or a cosmological constant(Paul J . Steinhardt and Neil Turok 2006). A cosmological constant has the effect of pulling and clumping objects together with gravity as a repulsive force. On the other side, while the Universe expands, the gravitational attraction between objects decreases, and the cosmological repulsion increases. The only visible proof of the expansion is the redshift of the disappearance of distant galaxies from the Milky Way. Milky Way galaxy -depends on some of the observers- does not expand. However, one of the closest galaxies around us, the Andromeda galaxy, moves fast enough to collide with our galaxy in 3 billion years. The expansion of the Universe can be exampled with the raisin bread model, as shown in Error! Reference source not found.. (Misner, Thorne, and Wheeler 1973) gave one example to show how the Universe expands within the local systems. Their example is the situation of pennies attached to a balloon's surface. The distance between pennies (their sizes remain same) inside the balloon increases when the balloon expands.

Another example is that the bread can be defined as a universe in this animation example, and raisins can be defined as galaxies or stars' clusters. While expanding during baking, raisins will not expand; however, the raisins will move away from each other. Some observers agree that galaxies and local systems are not expanding in an expanding Universe, while others agree to expand the local systems. 


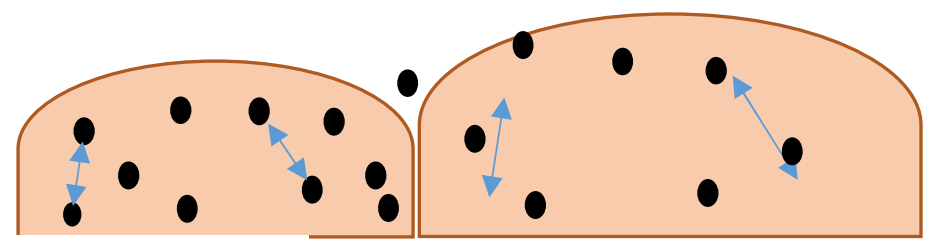

(b) Befor

(a) After

Figure 1. The illustration shows an expanding raisin bread model. In this model, while bread expands, only the distance between raisins expands, not raisins.

The human being did not know the rest of the galaxies around us before a century. From today's perspective, starting with the "Big Bang," billions of galaxies and stars are moving away from each other as the cosmic space expands. Hubble saw the sky and realized that galaxies were moving away from us. The farther galaxies were moving faster than the closest ones. He proved his observation and announced that the Universe was expanding at sure of speed. Figure 2 presents Hubble's graph for nearby galaxies, becoming a scientific landmark in astronomy textbooks. A linear relation between the galaxy's velocity(v) vs. distance(d) can be seen on the graph.

$v=H_{0} x d$

Equation 1

The slope of the relation, $H_{0}$ is the Hubble Constant, which is the cosmic expansion's constant rate caused by expanding spacetime itself. $1 / \mathrm{H}_{0}$ is the Hubble time.

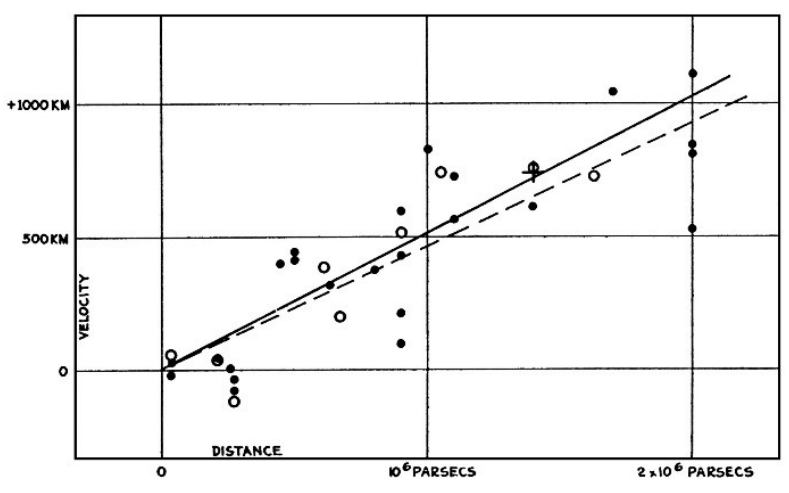

Figure 2. Velocity-Distance Relation among Extra-Galactic Nebulae (Edwin Hubble 1929).

Three steps for the calculation of Universe expansion' speed can be seen on the Hubble Constant value in Error! Reference source not found. over time. First, a cosmic distance ladder is a series of measurements of several objects built to measure exact distances to the galaxies from nearby to farther away. Second, researchers use the cosmic distance ladder to calculate the distances with intrinsic brightness. 


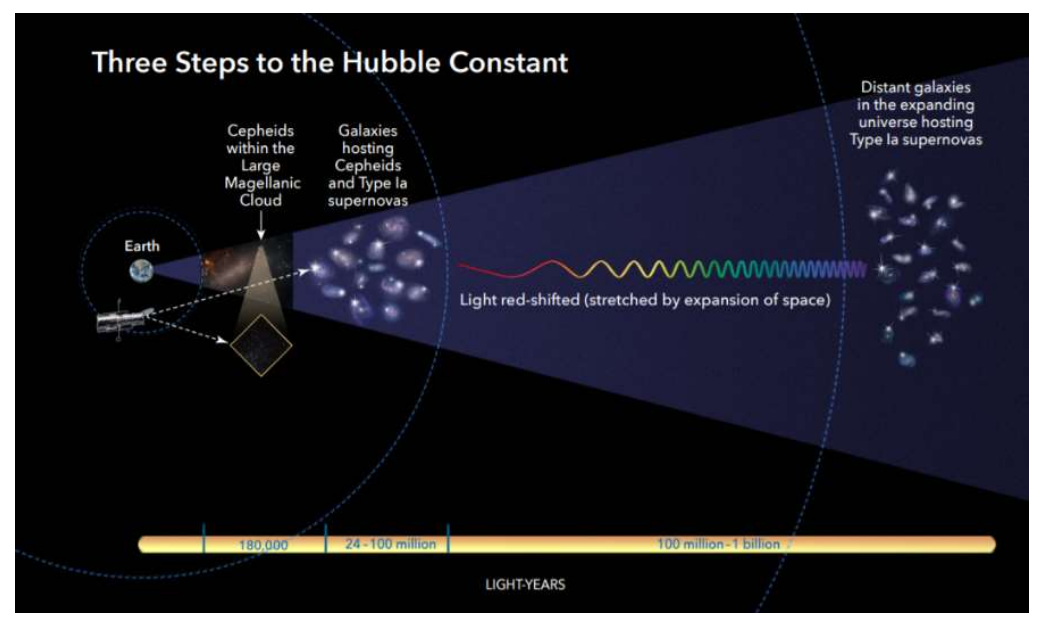

Figure 3. An illustration of three steps to the Hubble constant (NASA 2019).

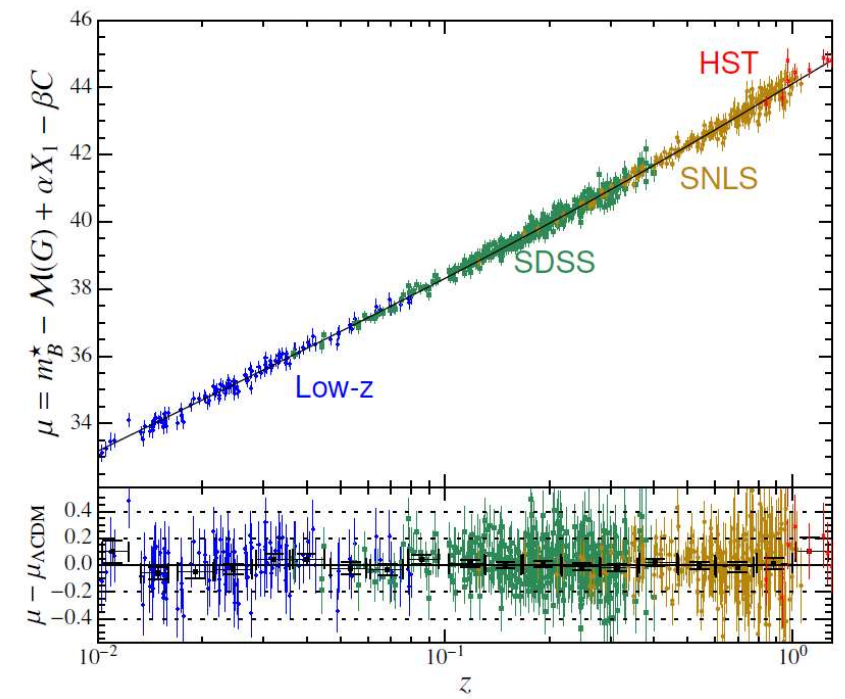

Figure 4. Top: Hubble diagram of the joint sample. Bottom: Residuals from the best-fit $\Lambda$ CDM cosmology as a redshift function (Betoule et al. 2014).

Error! Reference source not found. presents the observed Hubble Diagram's compiling using standard candles (SNIa) for the greater distances than Hubble observed. Hubble's values for his distances in 1929 were 500 $\mathrm{km} / \mathrm{s} / \mathrm{Mpc}$, a large factor of 7 . Today's calculations are less than $70 \mathrm{~km} / \mathrm{s} / \mathrm{Mpc}$ $\left(1 \mathrm{Mpc}=10^{6}\right.$ parsec $=3.26 \times 10^{6}$ light year $)$. The megaparsec $(\mathrm{Mpc})$ is telling us that galaxies -3.3 million light-years farther away from us- are moving away from us $70 \mathrm{~km}$ per second faster due to the expansion of the Universe. Hubble concluded that Andromeda is separate galaxies from our galaxy by using Cepheids and Leavitt's law. His conclusion showed that Milky Way is not the whole Universe for the first time. Hubble used Cepheids law to infer the distances to nearby galaxies. In contrast to their speeds, the graph showed cosmic expansion (Kirshner 2004; Bahcall 2015). 
Many researchers created the theories and studied after his statement in cosmology. One of the theories that became dominant in cosmology is the big bang model. Cosmology is a study of the overall structure of the Universe. The Universe is homogeneous according to the big bang model. As far as we can look at space in billions of light-years directions, we see the same stars, galaxies, or other materials in each direction. According to General Relativity, the Universe depends on density, whether infinite or finite. Depends on the size of the Universe, we can come up with a couple of ideas about the expansion, which can be represented in Error! Reference source not found. (Wright 2014).

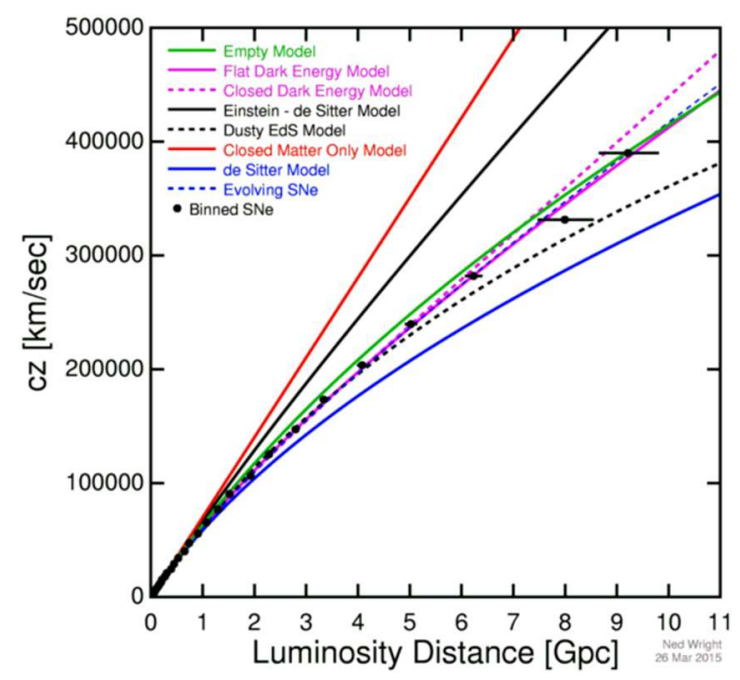

Figure 5. The curves show a closed Universe $(\Omega=2)$ in red, the critical density Universe $(\Omega=1)$ in black, the empty Universe $(\Omega=0)$ in green, the steady-state model in blue, and the WMAP based concordance model with $\Omega \mathrm{M}=0.27$ and $\Omega \mathrm{V}=0.73$ in purple (Wright 2014).

This model, which gives $\mathrm{H}_{\mathrm{o}}=71 \mathrm{~km} / \mathrm{sec} / \mathrm{Mpc}$, is used to level the plot's luminosity distances by showing a Universe's speed at low to medium redshifts. A slowing Universe at higher redshifts is a steady model with a cosmological constant and a significant dark matter (Wright 2014).

Measurements of the Universe give us data to find the density of the Universe. However, the expansion of the Universe can be questionable with the infinite or finite. If the Universe is infinite, how we can say that the Universe expands. Observers expected that expansion should not affect local systems such as atoms, solar, and galaxies. We cannot observe the expansion if everything expands at the same rate. Therefore, the Universe should be finite, and then we can develop the idea of expansion. On the other hand, even in the infinite Universe, we can ask another question about the galaxies and our solar systems. Are they expanding even in the infinite Universe?

Scientists have been studied the global cosmological expansion's effects on the local gravitating systems for a long time. Researchers focus on cosmological expansion and its effects on local systems used in theoretically important ways in the literature. The agreement is that there is an effect in impression undetectably small. In the present paper, we revisited and 
addressed this expansion of local systems question using some earlier assumptions. We studied whether there is an expansion in our local system in better agreement with the existing cosmological image. This article investigated the expansion and acceleration of the solar system, which engages today's flourishing cosmology field.

\section{2-. Discussion}

In this section, the cosmological expansion's effects on the local system's dynamics are discussed from different observers' perspectives, from negative to positive.

In the beginning, there was not any Universe. Billions of years ago, Universe started with the Big Bang or explained other theories and formed. Thousands of years from our day, cosmologists have been questioning how the Universe happened. Especially last hundred years, many researchers and cosmologists have been searching for this answer. However, researchers have been able to piece together a rough timeline of noteworthy events in the cosmos' life and its expansion using telescope observations and particle physics models. Every second, the Universe is getting much bigger. The space between galaxies extends as dough rises in the oven or pennies attached to the expanding balloon. However, how fast is the Universe expanding, and how this expansion affects the local systems? As Hubble and other telescopes look to answer these questions, they have a fascinating variation between what scientists and observers foresee and what they observe.

The Universe's general expansion had some effect on the evolution of the galaxy's solar system and the galaxy. Some papers argue that general relativity implies an essential connection between local systems and the rest of the Universe, and some of the arguments are against these arguments. Several authors have previously expressed that general relativity predicts no appreciable effect on the solar system due to the expansion of the Universe. Researchers studied the solar system scale's cosmic expansion affects more than 50 years ago. (De Sitter 1930) derived equations of motion and a star in the expanding Universe. According to his equations, the integral of areas is not affected by the expansion of the Universe. It means that the orbits of the stars stay ellipses, and the galaxies do not expand. Cosmological expansion studies help us understand how it affects the local systems and how the dynamics of surrounding non-symmetric matter for calculating the local environment's effect on the dynamics of the system of interest.

(McVittie 1933) calculated the expansion on planetary orbits and concluded that the orbital radius remains constant for a researcher using coordinates fixed in the solar system. This problem was taken by (Järnefelt $1942,1940)$ and reanalyzed and confirmed that the planetary orbits do not take part in the expansion of the Universe. (Einstein and Straus 1946) adopted an entirely diverse method and concluded that the planetary orbits do not expand with the Schwarzschild solution, which could be entrenched in a dust-expanding universe.

According to the Schwarzschild vacuum region model, a spherically symmetric compact object is surrounded by a vacuum inside a spatially homogeneous, test particles (presented the planets in local systems) never affected by the expansion. (Gott III et al. 2005) and the isotropic Universe (Nolan 1999). (Florides and W.H.McCrea 1959; Godfredsen 1961) stated that 
the solar system and cluster of galaxies are not affected by cosmological expansion. (David Layzer 1962) (Schücking 1954) evaluated a small correction to clock rates in coordinate time. The size of a bound system (Solar System, galaxy, or a cluster of galaxies) in an otherwise empty cavity is fixed so long as the system is isolated.

(R H Dicke 1964) used general arguments of particles in orbits are unaffected by the expansion. They showed that the orbit's coordinate radius decreases as the Universe of the scale factor and radius remain constant. (Misner, Thorne, and Wheeler 1973) gave a classical representation for the expansion of the Universe in their "Gravitation" book. The representation is a universe modeled by a rubber balloon's surface covered with coins (coins are galaxies). As the balloon inflates, coins are not stretching, but the distance between the coins stretch. (James L. Anderson 1995) et al. used Einstein, infield, and Hoffmann to study the cosmic expansion's action on local systems in an Einstein-de Sitter Universe and concluded that expansion does not affect if they are in a circular orbit. His theory did not give a clear statement of the scale of the local system expansions. Einstein-de Sitter model is a developing Friedmann model, while the initial de Sitter effect is ahistorical inquisitiveness. (Bonnor 1996) et al. modeled a relationship between the mass of the central particle, cosmic density, and central mass of the galaxies cluster and reached no orbits' expansion. (Rees 2005) et al. explained that space expands and carries objects with it. Neither planets nor any other objects in space do not expand. (Senovilla and Vera 1997) et al. did not have any solution with the cylindrical analog of the Einstein-Straus problem about the local system expansions.

After these negative results, it is our connection that this is incorrect. After Hubble's expanded-Universe study by using the cosmological redshift, scientists started to work on how the expansion affecting on the local system. (Peter D. Noerdlinger 1971) et al. found that the evolution of bound systems' sizes depends strongly on the cosmic fluid's energy density of zero rest mass and hardly on the cluster's first size. Clusters would expand only with a rate that alters the ratio of their density to the cosmic density. They used McVittie's model to study clusters of galaxies in the cosmic expansion. In their calculations, if the energy density $\left(\rho c^{2}\right)$ of particles with zero rest mass is less than $\left(\rho_{0} c^{2}\right)$ (the mean density of cold matter in the bound system), then the effect of the expanding Universe is to produce a small expansion of the bound system, with an equivalent Hubble constant. Researchers studied the two-body problem inside a cluster. In dust dominated F.R.W. universe, the average orbital radius's time derivative equation is given by

$<\dot{r}>=\frac{3 \epsilon}{1+4 \epsilon} H<r>$

Equation 2

( $\epsilon$ is the ratio of average energy densities in the cluster.) The cosmic expansion is crucial for systems whose sizes and lifetime become affiliated to Hubble radius and times. A Hubble constant is a number that denotes how fast the Universe is expanding.

(R H Dicke 1964; Peter D. Noerdlinger 1971; James L. Anderson 1995; Cooperstock, Faraoni, and Vollick 1998; Bonnor 2000) et al. are considered the 
shortened model of the local system entrenched on an R.W. picture, which encompasses two critical assumptions dissimilar to modern cosmological models: the Einstein equations and the gravity. (Dominguez and Gaite 2001) studied the Newtonian Equation of motion of the particles developing the small-scale constrained system.

$$
m \frac{d^{2} r_{i}}{d t^{2}}=F i(r)-m \emptyset, i(r, t)
$$

\section{Equation 3}

(The position of any particle $(r)$; internal forces between particles within the system $\left(\mathrm{F}_{\mathrm{i}}\right)$; gravitational potential $\left.(\varnothing i(r, t))\right)$. They used the RobertsonWalker (R.W.) metric to see if enormous-size expansion indicates a time dependence of the small systems volume. They carried out their study in the Newtonian limit of General Relativity to provide a clear and simple explanation of how the cosmological expansion may affect small systems. They concluded that the Universe's expansion affects small systems, but it is minimal to be ignored. (Gautreau 1984) embedded a particle in a heterogeneous and pressure-free expanding universe. He studied the influence of expansion on planetary orbits for analyzing geodesics. He worked with curvature coordinates $(R, T)$, in terms of which the metric has the form

$$
d s^{2}(R, T)=A^{-1}(R, T) d R^{2}+R^{2} d \Omega^{2}-B(R, T) d T^{2} \quad \text { Equation } 4
$$

In his model showed that the orbits expand. (Cooperstock, Faraoni, and Vollick 1998) have done another negligible study on local systems using Fermi normal coordinates. The metric in F.R.W. coordinates for an Einsteinde Sitter Universe is given by

$$
d s^{2}=-d t^{2}+a(t)^{2}\left(d x^{2}+d y^{2}+d z^{2}\right) \quad \text { Equation } 5
$$

$a(t)$ is the scale factor using Fermi's usual coordinates. The equation of motion is given by,

$$
\frac{d^{2} x_{F}^{k}}{d \tau^{2}}-\left(\frac{\ddot{a}}{a}\right) x_{F}^{k}=0
$$

Equation 6

The effects of the Universe's expansion on local systems' dynamics are exemplified by the corrections induced in the two-body problem (McVittie 1933; R H Dicke 1964; Peter D. Noerdlinger 1971; James L. Anderson 1995). According to the authors, all systems are affected by the expansion, but the cumulative effect of cosmological expansion on the Sun-Earth system's radius and angular motion is negligible. (Bonnor 2000) used FriedmannRobertson-Walker (F.R.W.) universe without any spatial curvature on the expansion of hydrogen atoms. He used the circumstantial Maxwell equations 
and established the Bohr model in its ground state within the Sitter Universe. The circular electron orbits in the de Sitter Universe remain unchanged during the expansion. F.R.W. universe equation with no spatial curvature:

$d s^{2}=-[R(t)]^{2}\left(d r^{2}+r^{2}\left(d \theta^{2}+\sin \theta^{2} d \emptyset^{2}\right)\right)+c^{2} d t^{2}$ Equation 7

(A dimensionless function, $R$; the speed of the light, c.) Maxwell equations on this metric:

$$
\begin{aligned}
& F^{i k}=K_{i ; k}-K_{k ; i}, \\
& \left(F^{i k}\right) ; k=4 \pi J^{i}
\end{aligned}
$$

(The electromagnetic field tensor, Fik; the potential, $\mathrm{K}_{\mathrm{i}}$; the current, and $\mathrm{J}^{\mathrm{i}}$.) He completed his calculations as below:

$\frac{4 \pi^{2} a^{2}}{9 A\left(c t_{0}\right)^{2}} \sim 10^{-67}$ in cgs units

Equation 10

He concluded that the atom expands in the Einstein-de Sitter universe in estimate calculations, but a rate is too low to trivial compared with the common cosmic expansion. A model is used for spacetime in another work, a solid solution of Einstein's Equation being a gravitating body in an asymptotically expanding Universe. Their model's calculations used geodesics in a McVittie metric and an asymptotically Robertson-Walker (R.W.) spacetime. According to their work, a local system can only feel the cosmological expansion if it is not spherically symmetric and has matter within the local system (Bolen, Bombelli, and Puzio 2001). After one year later, (Bonnor 2000) reconsidered his early work in the Einstein-Straus model. A group of researchers collected micrometeorites smaller than $1 \mathrm{~mm}$ from various environments such as deep-sea sediments and polar caps. They checked some elements such as Oxygen, $\mathrm{Fe}, \mathrm{Mn}$, An to show as they were an expanding solar system's inventory (M. Gounelle, C. Engrand, M. Chaussidon 2005). (Sereno and Jetzer 2007) opened a new crucial point related to the expansion of the planetary orbits. They concluded that planetary orbits are expanding, but the orbital properties' effects depend on the expansion's acceleration rather than on the expansion itself. (Pacheco 2015) re-studied the possible effects of the expansion on bonded systems. Expansion effects are unnoticeable so that they can be neglected in atomic and solar system scales.

$\frac{1}{A} \frac{d A}{d t}=\frac{P^{2}}{4 \pi^{2}}\left(1+3 e^{2}\right) \frac{d}{d t}\left(\frac{\ddot{a}}{a}\right)$

Equation 11 
(Pacheco 2015) showed that equation (6) shows that the main factor of expansion effects on the local system's orbit is the acceleration of the expansion with agreement from the previous conclusion (Sereno and Jetzer 2007). Pacheco generally agreed that the expansion rates are relatively low and can be neglected for all practical purposes. It is specified that the local interactions and global expansion effects exist on small cosmological scales with the relationship between Hubble distance and redshift. (Bolen, Bombelli, and Puzio 2001) obtained a first rough evaluation of the amount of these effects by evaluating $\boldsymbol{\sigma}$ and $\Delta \varepsilon / \varepsilon$ for a few known systems. For the solar planets Mercury and Pluto, Hubble parameter value $\mathrm{H}_{0}$ of around $75 \mathrm{~km} \mathrm{~s}^{-1}$ $\mathrm{Mpc}^{-1}$ for $\dot{\beta}^{0}$, one acquires for the comparative size of the expansion which in terms of precession.

$\sigma \approx \frac{6 \pi G M}{a\left(1-\varepsilon^{2}\right) c^{2}}+\frac{3 \pi}{4} \frac{\dot{\beta_{0}^{2}} a^{3}\left(1-\varepsilon^{2}\right)^{3}}{G \cdot M .}$

Equation 12

values of $\frac{\sigma_{\beta}}{\sigma_{0}} \approx 3 \times 10^{-21}$ and $7 \times 10^{-1}$, respectively, and for the relative eccentricity change in

$$
\left.\frac{\Delta \varepsilon}{\varepsilon} \approx-\frac{\pi G M \beta_{0} m}{2 c^{2} L u_{0}}=-\frac{\pi \dot{\beta}_{0}}{2 c^{2}} \sqrt{G M a\left(1-\varepsilon^{2}\right.}\right) \quad \text { Equation } 13
$$

values of $\frac{\Delta \varepsilon}{\varepsilon} \approx \frac{-1.5 \times 10^{-2}}{\text { rev }}$ and $\frac{-1.5 \times 10^{-2}}{r e v}$, respectively.

They also studied binary pulsar (Karastergiou et al. 2000) and stellar motion around the galactic center (Fragile and Mathews 2000)under general relativistic effects. They took the spherical universe model for their calculations. Their calculations concluded that all expansion effects are small and trivial for astrophysics. (Linder 2003) took the flat Universe model for his calculations. He used type Ia supernovae and standardized candles to map the expansion history $a(t)$ since there exists a direct relationship between the observed distance-redshift relation $d(z)=(1+z) \eta(z)$ and the theoretical $a(t)$.

$$
\eta(z)=\int_{a_{e}}^{1} \frac{d a}{a^{2} H}=\int_{0}^{z} d z^{\prime} / H\left(z^{\prime}\right)
$$

Equation 14

$\eta$ is the conformal time, $H=a \dot{a} a$ is the Hubble parameter, $a_{e}=\frac{1}{1+z}$ is the scale factor of the supernova exploration emission in a flat Universe model.

$$
t(a)=\int_{0}^{1} \frac{d a^{\prime}}{a^{\prime} H}=\int_{0}^{z} \frac{d z^{\prime}}{\left[\left(1+z^{\prime}\right) H\left(z^{\prime}\right)\right]}
$$

Equation 15

The early Universe's thermal history extended our physical understanding by learning about cosmology, astrophysics, and particle 
physics. (Noerdlinger 2008) studied the expansion of the solar system by using solar mass loss. Depend on Noerdlinger's work, the radiative and particulate loss of mass by the Sun, $\frac{\dot{M}_{0}}{M_{0}}=-9.13 \times 10^{-14} y^{-1}$ or more causes the orbits of the planets to expand at the same rate. The energy-matter's gravitational components in the Universe can affect a planetary system's orbit. In a dark matter Universe, (Pitjeva and Pitjev 2013), a gravitational system expands or contracts according to the amount and equation of the dark energy state. According to the $\Lambda \mathrm{CDM}$ scenario, the solar system should be expanding if we consider only the cosmological background's effect. (Sereno and Jetzer 2007) used Robertson Walker's (R.W.) metric to investigate the evolution of a planet's orbit around a massive star embedded in a homogeneous and isotropic universe. Finally, a recent study in 2018 by (Genova et al. 2018) studied the solar system expansion using NASA Messenger Data. It concluded that their analysis enhances the relativistic parameter's knowledge and confirmed Einstein's General Relativity theory. Their results showed that the solar system expands due to the solar mass loss.

Hundreds of researchers are searching for precise answers to how global expansion affects local systems every year. Every one of them uses different methods to calculate the expansion if there is a solar expansion. As we reviewed the literature in this section, besides non-believers with their negative results, more observers concluded that the local systems are expanding. Therefore, we will revisit one of the latest works with an updated Sun's luminosity data to calculate the solar system expansion.

\section{Analysis and Results}

An enormous amount of energy is releasing from the stars (i.e., Sun) into space. Indeed, life on Earth would not exist without the light from the Sun that powers it. We used Kepler's Third Law from orbital mechanics to revisit Reed's calculations (Reed and Vannette 2018).

$$
\begin{aligned}
& r=\left(\frac{G M T^{2}}{4 \pi^{2}}\right)^{1 / 3} \\
& \frac{r^{3}}{M}=\left(\frac{G T^{2}}{4 \pi^{2}}\right)
\end{aligned}
$$

Equation 16

Equation 17

$\mathrm{M}=1.99 \times 10^{30} \mathrm{~kg}$ is the mass of Sun, $T$ is the Earth's orbital period, and $\left(r=1.50 \times 10^{11} \mathrm{~m}\right)$ is the orbital radius. As an assumption for the calculation, the Earth, and Sun is an isolated system. We believe that the Earth's orbital radius will increase in the orbital radius due to decreasing the gravitational force by decreasing the Sun's mass. Once we took the time-derivative $\frac{d r}{d t}$ of Equation 16, the new formula is given,

$$
\frac{d r}{d t}=\frac{1}{3}\left(\frac{G M T^{2}}{4 \pi^{2}}\right)^{-2 / 3}\left(\frac{G T^{2}}{4 \pi^{2}}\right)\left(\frac{d M}{d t}\right)
$$


We substituted Equation 17 into Equation 18, and the new equation will be given,

$$
\frac{d r}{d t}=\frac{1}{3}\left(\frac{r}{M}\right)\left(\frac{d M}{d t}\right)
$$

Equation 19

The Sun's luminosity derives from the conversion of mass into energy in its core through fusion reactions. With the $E=M c^{2}$ equation, $c=$ $3.00 \times 10^{8} \mathrm{~m}$, the power output $\mathrm{P}$ of the Sun is then given,

$$
P=-\frac{d E}{d t}=-\left(\frac{d M}{d t}\right) c^{2}=3.830 \times 10^{26} \mathrm{~W}
$$

Equation 20

In this calculation, Reed assumed that the Earth is circulating in orbit all the time. This calculation will be negative in the current assumption, which means the sun and earth orbit radius decreases. In this equation, we took negative value due to Sun's mass losing expectation because Sun must lose its mass to create positive energy output, which is $\frac{d M}{d t}<0$. The radius of the Earth's orbit will be $\frac{d r}{d t}=0.1069 \times 10^{-9} \frac{\mathrm{m}}{\mathrm{s}} \sim-1.1 \times 10^{-1} \frac{\mathrm{m}}{\mathrm{s}}$ or about $-0.1 \frac{\mathrm{nm}}{\mathrm{s}}$.

However, in reality, Earth's circular orbit of the radius is changing. With an angular momentum of the Earth, $m=5.972 \times 10^{24} \mathrm{~kg}$ is the Earth-mass, $r=1.5 \times 10^{8} \mathrm{~km}$ is the Earth's circular orbit of radius, $v$ is the Earth's speed. The angular momentum of Earth is given $(L=m * v * r)$. Reed et al. assumed the speed; the Sun's gravitational attraction causes the centripetal force keeping the Earth in its orbit;

$\frac{G * M * m}{r^{2}}=\frac{m v^{2}}{r} \rightarrow \quad v=\sqrt{\frac{G M}{r}}$. When we substitute this equation in $(L=m *$ $v * r$ ), we will have

$$
(L=m * \sqrt{G * M * r})
$$

Equation 21

When we took the time-derivative of equation 20 , the final equation is given by,

$$
\frac{d L}{d t}=\frac{m \sqrt{G}}{2}\left[\sqrt{\frac{r}{M}\left(\frac{d M}{d t}\right)}+\sqrt{\frac{M}{r}\left(\frac{d r}{d t}\right)}\right]=0
$$

Equation 22 will become zero because $L$ must be conserved.

$$
\frac{m \sqrt{G}}{2} \sqrt{\frac{r}{M}\left(\frac{d M}{d t}\right)}=\frac{m \sqrt{G}}{2} \sqrt{\frac{M}{r}\left(\frac{d r}{d t}\right)} \rightarrow \frac{d r}{d t}=-\left(\frac{r}{M}\right)\left(\frac{d M}{d t}\right) \quad \text { Equation } 23
$$


Substituting the $\frac{d M}{d t}$ from Equation 20 into equation 23 gives

$\frac{d r}{d t}=\frac{r P}{M c^{2}}$

Equation 24

Once we calculate equation $24, \frac{d r}{d t}=0.3207 \times 10^{-9} \frac{\mathrm{m}}{\mathrm{s}} \sim 3.2 \times 10^{-10} \frac{\mathrm{m}}{\mathrm{s}}$ or about
$0.3 \frac{\mathrm{nm}}{\mathrm{s}}$.

\section{Conclusion}

This paper reviewed the Cosmological effect on the local system in literature and revisited Reed's calculations. The researchers focused and measuring methods such as the planet's and galaxies' relative distances and Sun-Earth distances. When we revisited Reed's calculation, we also showed Reed's work to understand the solar system expansion due to the solar mass loss in cosmological expansion.

With the value, the Earth and Sun distance, based on Kepler's third law, was 148.37 million $\mathrm{km}$, corresponding to $\left(3.2 \times 10^{-1} \frac{\mathrm{m}}{\mathrm{s}}\right)$, which is $\sim 1 \mathrm{~cm} /$ year compared to Suntola's (Suntola 2018) Earth and Moon expansion value 1.1 $\mathrm{cm} / \mathrm{y}$. Earth's orbit is affected by the expansion of the Universe. In agreement with Reed's calculations, we showed an expansion in the solar system under cosmological expansion by revisiting Reed's calculation. In conclusion, every small-scale system expands or contract in an expanding Universe at different rates.

\section{References}

Bahcall, Neta A. 2015. "Hubble's Law and the Expanding Universe." Proceedings of the National Academy of Sciences of the United States of America 112 (11): 3173-75. https://doi.org/10.1073/pnas.1424299112.

Betoule, M., R. Kessler, J. Guy, J. Mosher, D. Hardin, R. Biswas, P. Astier, et al. 2014. "Improved Cosmological Constraints from a Joint Analysis of the SDSS-II and SNLS Supernova Samples." Astronomy and Astrophysics 568. https://doi.org/10.1051/0004-6361/201423413.

Bolen, Brett, Luca Bombelli, and Raymond Puzio. 2001. "Expansion-Induced Contribution to the Precession of Binary Orbits." Classical and Quantum Gravity 18 (7): 1173-78. https://doi.org/10.1088/0264-9381/18/7/302.

Bonnor, W B. 1996. "The Cosmic Expansion and Local Dynamics." Mon. Not. R. Astron. Soc 1469: 1467-69.

Bonnor, W. B. 2000. "Local Dynamics and the Expansion of the Universe." General Relativity and Gravitation 32 (6): 1005-7. https://doi.org/10.1023/A:1001961325184.

Cooperstock, F. I., V. Faraoni, and D. N. Vollick. 1998. "The Influence of the Cosmological Expansion on Local Systems." The Astrophysical Journal 503 (1): 61-66. https://doi.org/10.1086/305956.

David Layzer. 1962. "A Praface to Cosmogony. I. The Energy Equation and the Virial Theorem for Cosmic Distributions." American Astronomical Society 1: 174-84.

Dominguez, A., and J. Gaite. 2001. "Influence of the Cosmological Expansion on Small Systems." Europhysics Letters 55 (4): 458-64. https://doi.org/10.1209/epl/i2001-00437-y.

Edwin Hubble. 1929. "A Relation between Distance and Radial Velocity among Extra-Galactic Nebulae." Proc. N.A.S. 15: 168-73.

Einstein, A., and E. G. Straus. 1946. "Corrections and Additional Remarks to Our Paper: The Influence of the Expansion of Space on the Gravitation Fields Surrounding the Individual Stars." Reviews of Modern Physics 18 (1): 148-49. https://doi.org/10.1103/RevModPhys.18.148.

Florides, P.S., and W.H.McCrea. 1959. "Observable Relations in Relativistic Cosmology III.” Springer-Verlag 48 (52-71).

Fragile, P. Chris, and Grant J. Mathews. 2000. "Reconstruction of Stellar Orbits Close to Sagittarius A*: Possibilities for Testing General Relativity." The Astrophysical Journal 542 (1): 328-33. https://doi.org/10.1086/309495.

Gaureau, Ronald. 1984. "Curvature Coordinates in Cosmology." Annals of the New York Academy of Sciences 422 (1): 351-351. https://doi.org/10.1111/j.1749-6632.1984.tb23382.x. 
Genova, Antonio, Erwan Mazarico, Sander Goossens, Frank G. Lemoine, Gregory A. Neumann, David E. Smith, and Maria T. Zuber. 2018. "Solar System Expansion and Strong Equivalence Principle as Seen by the NASA MESSENGER Mission." Nature Communications 9 (1). https://doi.org/10.1038/s41467-017-02558-1.

Godfredsen, Eugene A. 1961. "Remarks on the Dynamical Stability of Galaxy Clusters." The Astronomical Journal 70: 285.

Gott III, J. Richard, Mario Jurić, David Schlegel, Fiona Hoyle, Michael Vogeley, Max Tegmark, Neta Bahcall, and Jon Brinkmann. 2005. "A Map of the Universe." The Astrophysical Journal 624 (2): $463-84$. https://doi.org/10.1086/428890.

James L. Anderson. 1995. "Multiparticle Dynamics in an Expanding Universe.” Physical Review Letters 75 (20): 3602-4.

Järnefelt, G. 1940. “Järnefelt, G.” Ann. Acad. Sci. Fenn. Ser. A55 (3).

Järnefelt, 1942. "Järnefelt, G.” Ann. Acad. Sci. Fenn. Ser. A1 (12).

Karastergiou, A., M. Kramer, N. Wex, and A. von Hoensbroech. 2000. "Geodetic Precession and the Binary Pulsar B1913+16." International Astronomical Union Colloquium $177 \quad$ (1998): 125-26. https://doi.org/10.1017/s0252921100059236.

Kirshner, Robert P. 2004. "Hubble's Diagram and Cosmic Expansion” 101 (1).

Linder, Eric V. 2003. "Exploring the Expansion History of the Universe." Physical Review Letters 90 (9): 4. https://doi.org/10.1103/PhysRevLett.90.091301.

M. Gounelle, C. Engrand, M. Chaussidon, M.E. Zolensky and M. Maurette. 2005. "An Achondritic Micrometeorite from Antarctica: Expanding the Solar System Inventory of Basaltic Asteroids." Lunar and Planetary Science, $5-6$.

McVittie, G C. 1933. "The Mass-Particle in an Expanding Universe." Monthly Notices of the Royal Astronomical Society 93 (5): 325-39. https://doi.org/10.1093/mnras/93.5.325.

Misner, Charles W, Kip S Thorne, and John Archibald Wheeler. 1973. Gravitation. Macmillan.

NASA. 2019. "Mystery of the Universe's Expansion Rate Widens With New Hubble Data." Nasa.Gov. 2019.

Noerdlinger, Peter D., Vahe Petrosian. 1971. "The Effect Of Cosmological Expansion On Self-Gravitating Ensembles Of Particles.” The Astrophysical Journal 168 (1): 59-76.

Noerdlinger, Peter D. 2008. "Solar Mass Loss, the Astronomical Unit, and the Scale of the Solar System," no. Sikivie 2005: 1-31. http://arxiv.org/abs/0801.3807.

Nolan, Brien C. 1999. "A Point Mass in an Isotropic Universe: III. The Region R $\leq 2 \mathrm{~m}$." Classical and Quantum Gravity 16 (10): 3183-91. https://doi.org/10.1088/0264-9381/16/10/310.

Pacheco, José A.de Freitas. 2015. "What Expands in an Expanding Universe?" Anais Da Academia Brasileira de Ciencias 87 (4): 1915-27. https://doi.org/10.1590/0001-3765201520140650.

Paul J . Steinhardt and Neil Turok. 2006. "Why the Cosmological Constant Is Small and Positive." Science 312 (5777): 1180-83. https://www.jstor.org/stable/3846257.

Pitjeva, E. V., and N. P. Pitjev. 2013. "Relativistic Effects and Dark Matter in the Solar System from Observations of Planets and Spacecraft." Monthly Notices of the Royal Astronomical Society 432 (4): 3431-37. https://doi.org/10.1093/mnras/stt695.

R H Dicke, P J E Peebles. 1964. "EVOLUTION OF THE SOLAR SYSTEM AND THE EXPANSION OF THE UNIVERSE." Physical Review Letters 12 (1): 435-37.

Reed, B. Cameron, and Matthew Vannette. 2018. "Earth's Orbital Expansion Due to the Solar Luminosity: A Newton/Einstein Order-of-Magnitude Exercise." Physics Education 53 (5). https://doi.org/10.1088/1361$6552 /$ aad060.

Rees, Martin J. 2005. "Magnetic Fields in the Early Universe.” In Cosmic Magnetic Fields, 1-8. Springer.

Schücking, Engelbert. 1954. "Das Schwarzschildsche Linienelement Und Die Expansion Des Weltalls." Zeitschrift Für Physik 137 (5): 595-603.

Senovilla, José M.M., and Raül Vera. 1997. "Impossibility of the Cylindrically Symmetric Einstein-Straus Model." Physical Review Letters 78 (12): 2284-87. https://doi.org/10.1103/PhysRevLett.78.2284.

Sereno, Mauro, and Philippe Jetzer. 2007. "Evolution of Gravitational Orbits in the Expanding Universe" 064031: 1-8. https://doi.org/10.1103/PhysRevD.75.064031.

Sitter, W De. 1930. "On the Distances and Radial Velocities of Extra-Galactic Nebulae, and the Explanation of the Latter by the Relativity Theory of Inertia." Proceedings of the National Academy of Sciences of the United States of America 16 (7): 474.

Suntola, Tuomo. 2018. The Dynamic Universe Toward A Unified Picture Of Physical Reality.

Wright, Edward L. (Ned). 2014. "Measuring the Curvature of the Universe by Measuring the Curvature of the Hubble Diagram." http://www.astro.ucla.edu/ wright/sne_cosmology.html. 